\title{
Feasible Elimination Procedures in Social Choice: An Axiomatic Characterization
}

Citation for published version (APA):

Peleg, B., \& Peters, H. (2017). Feasible Elimination Procedures in Social Choice: An Axiomatic Characterization. Research in Economics, 71(1), 43-50. https://doi.org/10.1016/j.rie.2016.11.006

Document status and date:

Published: 01/03/2017

DOI:

10.1016/j.rie.2016.11.006

Document Version:

Publisher's PDF, also known as Version of record

Document license:

Taverne

Please check the document version of this publication:

- A submitted manuscript is the version of the article upon submission and before peer-review. There can be important differences between the submitted version and the official published version of record.

People interested in the research are advised to contact the author for the final version of the publication, or visit the DOI to the publisher's website.

- The final author version and the galley proof are versions of the publication after peer review.

- The final published version features the final layout of the paper including the volume, issue and page numbers.

Link to publication

\footnotetext{
General rights rights.

- You may freely distribute the URL identifying the publication in the public portal. please follow below link for the End User Agreement:

www.umlib.nl/taverne-license

Take down policy

If you believe that this document breaches copyright please contact us at:

repository@maastrichtuniversity.nl

providing details and we will investigate your claim.
}

Copyright and moral rights for the publications made accessible in the public portal are retained by the authors and/or other copyright owners and it is a condition of accessing publications that users recognise and abide by the legal requirements associated with these

- Users may download and print one copy of any publication from the public portal for the purpose of private study or research.

- You may not further distribute the material or use it for any profit-making activity or commercial gain

If the publication is distributed under the terms of Article $25 \mathrm{fa}$ of the Dutch Copyright Act, indicated by the "Taverne" license above, 


\title{
Feasible elimination procedures in social choice: An axiomatic characterization ${ }^{2 / 3}$
}

\author{
Bezalel Peleg ${ }^{a}$, Hans Peters ${ }^{\mathrm{b}, *}$ \\ a The Federmann Center for the Study of Rationality and the Institute of Mathematics, The Hebrew University of Jerusalem, Jerusalem \\ 91904, Israel \\ ${ }^{\mathrm{b}}$ Department of Quantitative Economics, Maastricht University, Maastricht, The Netherlands
}

\section{A R T I C L E I N F O}

\section{Article history:}

Received 16 October 2016

Accepted 26 November 2016

Available online 13 December 2016

Keywords:

Feasible elimination procedure

Anonymity

Maskin monotonicity

Independent blocking

Axiomatization

\begin{abstract}
A B S T R A C T
Feasible elimination procedures (Peleg, 1978) play a central role in constructing social choice functions which have the following property: in the associated game form, for any preference profile there exists a strong Nash equilibrium resulting in the sincere outcome. In this paper we provide an axiomatic characterization of the social choice correspondences resulting from applying feasible elimination procedures. The axioms are anonymity, Maskin monotonicity, and independent blocking. We also show that these axioms are logically independent.
\end{abstract}

(c) 2016 University of Venice. Published by Elsevier Ltd. All rights reserved.

\section{Introduction}

\subsection{Background}

We consider the classical social choice model with finitely many voters who have preferences - linear orderings - over a finite set of alternatives. A social choice function assigns an alternative to every profile of preferences, and induces an ordinal game in which every voter has the set of all preferences as strategy space, and evaluates an alternative according to his true, sincere preference. A social choice function is strategy-proof if reporting his sincere preference is a weakly dominant strategy for every player in this game. The theorem of Gibbard (1973) and Satterthwaite (1975) says that every strategy-proof social choice function with range at least three must be dictatorial on this range. In other words, any reasonable social choice function is manipulable.

Is this a serious problem? One may argue that strategic voting is an inherent property of democratic processes. For instance, it is a well-known phenomenon that in parliamentary elections people may not vote for their favorite party since such a vote may be lost if that party does not exceed the threshold, or is unlikely to become part of the government. Or one may argue that even though a social choice function is manipulable, it may still be difficult to manipulate successfully, due to limited information about the voting behavior of others or simply because of computational complexity (see for instance, Bartholdi et al. (1989), as an early reference). Nevertheless, attempts to manipulate or vote strategically are bound to result in an outcome that is not the sincere one - the outcome if voters vote truthfully - according to the social choice function under consideration. Assuming that this social choice function or voting system is chosen because of its appealing

\footnotetext{
Financial support from GSBE, Maastricht University, and of The Federmann Center for the Study of Rationality, The Hebrew University of Jerusalem, is gratefully acknowledged.

* Corresponding author.

E-mail addresses: pelegba@math.huji.ac.il (B. Peleg), h.peters@maastrichtuniversity.nl (H. Peters).
} 
properties, manipulation may result in a violation of such properties judged according to the true, sincere preferences. Therefore, it is desirable to attain the sincere outcome even if voters vote insincerely or strategically.

How can this be achieved? An equivalent way of formulating strategy-proofness is that the profile of sincere preferences is always a Nash equilibrium in the associated voting game. Since, according to the Gibbard-Satterthwaite theorem, strategy-proofness basically implies dictatorship, in effect strategy-proofness is equivalent to the profile of sincere preferences always being an even strong Nash equilibrium - no group of voters can profitably deviate. Peleg (1978) therefore considered the following weaker condition: for every profile of sincere preferences there exists a strong Nash equilibrium resulting in the sincere alternative, i.e., the alternative assigned if the voters would report sincerely. Social choice functions having this property are called exactly and strongly consistent (ESC). ${ }^{1}$ Thus, if an ESC social choice function is used, then it is at least plausible that the sincere alternative results.

One might object that the ESC property is too weak in the sense that there may be other strong Nash equilibria of the voting game not resulting in the sincere outcome. Unfortunately, however, requiring all strong Nash equilibria to result in the sincere outcome would again imply dictatorship of the social choice function - see e.g. (Peleg and Peters (2010), p. 100) for the argument. ${ }^{2}$ From this point of view, ESC is the best one can hope for. Moreover, even if a strong Nash equilibrium of the voting game does not result in the sincere outcome it is nevertheless an attractive outcome: it is not only Pareto optimal but a fortiori in the core of the voting game, which is just a somewhat different way of saying that no coalition can deviate to the satisfaction of all its members.

An additional motivation for using an ESC social choice function derives from the fact that then the sincere preference profile itself is not manipulable in the following sense. Suppose a coalition (group of voters) deviates from the sincere preference profile in such a way that all its members are better off by this deviation. Then there is a deviation by the complement of that coalition such that at least one member of the originally deviating coalition is not better off compared to the sincere outcome. In other words, by deviating from the sincere profile no coalition can guarantee (i.e., independently of the outside agents) to make all its members better off. See Peleg and Procaccia (2007), Peleg and Peters (2010), Sect. 8.5, and in a related context Peleg and Peters (2015). In still other words, ESC social choice functions not only admit a strong Nash equilibrium resulting in the sincere outcome but also make sincere voting itself a focal strategy for voters and coalitions in situations where it is difficult to predict how others will vote.

\subsection{Feasible elimination procedures}

ESC social choice functions can be obtained by selecting alternatives resulting from so-called feasible elimination procedures, introduced in Peleg (1978). A fortiori, using feasible elimination procedures is the only way to construct ESC social choice functions under three conditions, namely: the number of voters is greater than or equal to the number of alternatives minus one, the social choice function is anonymous, and it satisfies no veto power. The last condition says that no single agent can exclude any alternative from being chosen. See Peleg and Peters (2010), Sect. 10.5, which in turn is based on Holzman (1986). These conditions are natural and thus, feasible elimination procedures are essential for achieving this particular way of alleviating the consequences of the Gibbard-Satterthwaite Theorem.

Feasible elimination procedures work as follows. Assign weights (natural numbers) to the alternatives such that the sum of these weights is equal to the number of voters plus one. Consider a preference profile and delete an alternative $x$ that is bottom ranked by at least as many voters as the weight of $x$; also delete as many preferences from the profile, having $x$ bottom ranked, as the weight of $x$. Repeat this procedure for the reduced profile, until one alternative is left. This alternative is called 'maximal' for the preference profile under consideration. It does not have to be unique.

Could feasible elimination procedures be used in practice? To do so, voters should report complete preferences, but it is well recognized that eliciting more preference information than just (for instance) the top ranked alternatives is beneficial for the democratic process. In fact, systems applied in practice like plurality with runoff and in particular single transferable vote (STV) do use more preference information. Recently, Balinski and Laraki (2010) have proposed a voting system based on majority judgment, which asks extensive preference valuations from voters. Moreover, feasible elimination procedures, by definition, have the advantage of providing minorities of voters with the possibility of vetoing undesirable or unacceptable candidates. See Danilov and Sotskov (2002) for more discussion on this issue.

A potential drawback of feasible elimination procedures is that they may fail to be neutral: it is not always possible to choose all weights equal. However, if the number of voters is relatively large compared to the number of alternatives then this is only a slight problem, since weights can always be chosen such as to differ by at most one. Also, selecting from the set of maximal alternatives (the alternatives obtained from feasible elimination procedures) can be done in a neutral way. We refer the reader to Remark 2.3 for details.

Feasible elimination is a form of vetoing. Related veto procedures have been studied extensively in the literature, e.g. Dutta (1983), Moulin (1982), or Abdou (1989). Most of these veto procedures are based on a given ordering of the voters and they are therefore not anonymous, although they may be neutral. In general, social choice functions that are both neutral and anonymous only exist under certain relations between the number of voters and the number of alternatives (Moulin, 1980).

\footnotetext{
${ }^{1}$ In Moulin (1982) such a social choice function is called 'partially implementable'.

2 Briefly, such a social choice function implements itself in strong Nash equilibrium and therefore must be Maskin monotonic (Maskin, 1999). In turn, this implies dictatorship by Muller and Satterthwaite (1977).
} 


\subsection{Objective of this paper}

We have argued that ESC is a desirable property of a social choice function and that under a few natural conditions ESC social choice functions can only be obtained by selecting from maximal alternatives, i.e., alternatives resulting from feasible elimination procedures. Naturally, the correspondence of maximal alternatives associated with a given assignment of weights should have other reasonable properties besides leading to ESC social choice functions. Therefore, the objective of this paper is to provide an axiomatic characterization of these social choice correspondences. The axioms that we use are two general conditions: anonymity and Maskin monotonicity; and a condition related to the blocking power of coalitions. Anonymity is clear, and Maskin monotonicity (Maskin, 1999) says that if the position of a chosen alternative improves in the preference profile, then it will still be chosen in the new profile. Maskin monotonicity is well-known as a necessary condition for implementation.

For a given social choice function or correspondence, a coalition can block a set of alternatives if by putting this set at the bottom part of their preferences no assigned alternative will be in this set, whatever the reported preferences of the outside voters. A coalition minimally blocks such a set if no proper subcoalition can block it. The announced condition related to the blocking power of coalitions is called 'independent blocking'. Its main implication is that if two disjoint sets of alternatives are minimally blocked by two disjoint coalitions, then the union of the two sets of alternatives is minimally blocked by the union of those coalitions, that is, no proper subcoalition of that union can block the union of the two sets of alternatives. The main result of the paper is that the axioms of anonymity, Maskin monotonicity, and independent blocking characterize the social choice correspondences assigning the maximal alternatives, i.e., the alternatives resulting from feasible elimination procedures. We argue, furthermore, that this axiomatic characterization can be seen as one possible extension of the characterization of majority rule for the case of two alternatives by May (1952).

Section 2 presents preliminaries, and in Section 3 we state and prove the characterization result. Section 4 concludes.

Notations The following basic notations are used throughout. For a set $D,|D|$ denotes the cardinality of $D, P(D)$ the power set, i.e., the set of all subsets of $D$, and $P_{0}(D)$ the set of all nonempty subsets of $D$.

\section{Preliminaries}

Let $A$ be the set of $m$ alternatives, $m \geq 2$, and let $N=\{1, \ldots, n\}, n \geq 2$, be the set of voters. Subsets of $N$ are called coalitions. Let $L$ be the set of all preferences, i.e., complete, antisymmetric and transitive binary relations, on $A$. Then $L^{N}$ is the set of all (preference) profiles. A social choice correspondence (SCC) is a function $H: L^{N} \rightarrow P_{0}(A)$.

The main SCCs of interest in this paper are based on so-called feasible elimination procedures. Informally, first, assign weights $\beta(x) \in \mathbb{N}$ to the alternatives $x \in A$. Consider a preference profile and take an alternative $x$ that is bottom ranked at least $\beta(x)$ times. Delete that alternative from the profile and at the same time delete $\beta(x)$ preferences where $x$ is bottom ranked. Repeat this procedure until one alternative remains, which happens under appropriate conditions.

Formally, we have the following definition. (For a few examples see below.)

Definition 2.1. Assume that $n+1 \geq m$ and let $\beta: A \rightarrow \mathbb{N}$ satisfy $\sum_{x \in A} \beta(x)=n+1$. Let $R^{N} \in L^{N}$. A feasible elimination procedure (f. e.p.) for $R^{N}$ is a sequence $\left(x_{1}, C_{1} ; \ldots ; x_{m-1}, C_{m-1} ; x_{m}\right)$ such that

(a) $A=\left\{x_{1}, \ldots, x_{m}\right\}$,

(b) $C_{1}, \ldots, C_{m-1}$ are pairwise disjoint subsets of $N$ and $\left|C_{j}\right|=\beta\left(x_{j}\right)$ for all $j=1, \ldots, m-1$,

(c) $x_{k} R^{i} x_{j}$ for all $j=1, \ldots, m-1, k=j+1, \ldots, m$, and $i \in C_{j}$.

Thus, in a feasible elimination procedure $\left(x_{1}, C_{1} ; \ldots ; x_{m-1}, C_{m-1} ; x_{m}\right)$, by condition (c) alternative $x_{1}$ is bottom ranked for all voters in $C_{1}$ and by condition (b), $\left|C_{1}\right|=\beta\left(x_{1}\right)$. Now $x_{1}$ is deleted from $R^{N}$ and also the preferences of the voters in $C_{1}$ are deleted. In the remaining profile, $x_{2}$ is bottom ranked for all voters in $C_{2}$ by condition (c), and by condition (b), $\left|C_{2}\right|=\beta\left(x_{2}\right)$, so that $x_{2}$ can be deleted and also the preferences of the voters in $C_{2}$. And so on and so forth. Observe that after deleting $x_{1}$ there are $n-\beta\left(x_{1}\right)$ voters left, after deleting $x_{2}$ there are $n-\beta\left(x_{1}\right)-\beta\left(x_{2}\right)$ voters left, and after deleting $x_{\mathrm{m}-1}$ there are $n-\beta\left(x_{1}\right)-\ldots-\beta\left(x_{m-1}\right)=\beta\left(x_{m}\right)-1 \geq 0$ voters left.

An important observation about f.e.p.'s is the following. Suppose an alternative $x$ is bottom ranked by (at least) the voters in some coalition $S$ with $|S|=\beta(x)$, in a profile $R^{N} \in L^{N}$. Then $x$ must be eliminated in every f.e.p. for $R^{N}$. To see this suppose there is an f.e.p. in which $x$ is not eliminated and let $y$ be the alternative eliminated last, say via coalition $T$. Then the finally left voters form a coalition $S^{\prime}$ containing $S$. We have $\beta(y)+\beta(x)=|T|+\left|S^{\prime}\right|+1$ by the foregoing, but also $|T|+\left|S^{\prime}\right| \geq \beta(y)+\beta(x)$, a contradiction.

It is not difficult to see that there exists always at least one f.e.p. under the assumptions in the definition. If every alternative $x_{j}$ is bottom ranked less than $\beta\left(x_{j}\right)$ times, then the total number of voters is at most $\sum_{j=1}^{m} \beta\left(x_{j}\right)-m$, which is equal to $n+1-m$ and therefore strictly smaller than $n$. A similar argument can be made after elimination of each alternative $x_{1}, \ldots, x_{m-2}$.

Henceforth in this paper we assume $n+1 \geq m$. An alternative $x$ is $R^{N}-$ maximal if there exists an f.e.p. $\left(x_{1}, C_{1} ; \ldots ; x_{m-1}, C_{m-1} ; x\right)$. We denote

$$
M_{\beta}\left(R^{N}\right)=\left\{x \in A: x i s R^{N}-\text { maximal }\right\} .
$$


Thus, $M_{\beta}$ depends on the exogenously chosen weights $\beta(x) \in \mathbb{N}, x \in A$, which can be varied as long as $\sum_{x \in A} \beta(x)=n+1$.

The importance of the SCC $M_{\beta}$ derives from the fact that every selection from it produces an ESC social choice function, and that under some additional but natural conditions, selecting from $M_{\beta}$ for some weight function $\beta$ is the only way to achieve ESC social choice functions. We refer to Section 1 of the paper for more explanation.

Example 2.2. Let $A=\{a, b, c\}$.

(a) Let $N=\{1, \ldots, 5\}, \beta(a)=\beta(b)=\beta(c)=2$, and consider the preference profile

$\begin{array}{rrrrr}R^{1} & R^{2} & R^{3} & R^{4} & R^{5} \\ b & c & a & c & a \\ c & b & b & a & c \\ a & a & c & b & b\end{array}$

then there are two feasible elimination procedures, namely $(a,\{1,2\} ; b,\{4,5\} ; c)$ and $(b,\{4,5\} ; a,\{1,2\} ; c)$. Hence $M_{\beta}\left(R^{N}\right)=\{c\}$.

(b) Let $N=\{1, \ldots, 11\}, \beta(a)=\beta(b)=\beta(c)=4$, and consider the preference profile

$\begin{array}{cccc}R^{1}, R^{2}, R^{3} & R^{4}, R^{5} & R^{6}, R^{7}, R^{8} & R^{9}, R^{10}, R^{11} \\ b & c & a & a \\ c & b & b & c \\ a & a & c & b\end{array}$

In this case there are several feasible elimination procedures, due to the fact that there are several choices in selecting eliminating coalitions, but $a$ is always eliminated first. Two examples are: $(a,\{1,2,3,4\} ; b,\{5,9,10,11\} ; c)$ and $(a,\{1,2,4,5\} ; c,\{3,6,7,8\} ; b)$. In this case $M_{\beta}\left(R^{N}\right)=\{b, c\}$. Note that in this profile $a$ is the Condorcet and also the plurality winner, but it is also the alternative ranked last by a plurality of voters. In this example, the alternatives $b$ and $c$ may indeed be better 'compromises' than alternative $a$.

Note that it is not always possible to choose all weights equal as in the examples above, so it is not always possible to achieve neutrality.

Remark 2.3. In fact, there are two issues concerning neutrality. First, weights cannot always be chosen to be equal, but they can be chosen so as to differ by at most one. ${ }^{3}$ If the number of voters is relatively large then neutrality is almost achieved. For instance, if there are ten alternatives and thousand voters then nine weights can be chosen equal to 100 and the remaining weight equal to 101. Second, although $M_{\beta}$ may not be neutral, one can choose neutrally from $M_{\beta}\left(R^{N}\right)$.

\section{Axiomatic characterization of $M_{\beta}$}

Let $H$ be an SCC. We first introduce some terminology and notations concerning the blocking power of coalitions.

A coalition $S \in P_{0}(N)$ blocks a set $B \in P_{0}(A)$ at $H$ if for any profile $Q^{S} \in L^{S}$ for $S$ such that $x Q^{i} y$ for each $x \in A \backslash B$ and $y \in B$, we have $H\left(Q^{S}, R^{N \backslash S}\right) \subseteq A \backslash B$ for all $R^{N \backslash S} \in L^{N \backslash S}$. In words, a coalition blocks a set of alternatives if by putting this set at the bottom part of the preference of each coalition member, no alternative in this set will be chosen.

Coalition $S \in P_{0}(N)$ minimally blocks $B$ at $H$ if no proper nonempty subcoalition of $S$ blocks $B$ at $H$. If a set $B \in P_{0}(A)$ is blocked by some nonempty coalition at $H$, then we define the blocking coefficient $b_{H}(B)$ as the minimal size of such a nonempty coalition, hence

$$
b_{H}(B)=\min \left\{|S|: S \in P_{0}(N) \text { blocks } B \text { at } H\right\} .
$$

Otherwise, we define $b_{H}(B)=n+1$. Note that, since $H\left(R^{N}\right) \neq \varnothing$ for all $R^{N} \in L^{N}$ by definition, no coalition blocks $A$, and thus $b_{H}(A)=n+1$ always. The choice of blocking coefficient $n+1$ for an unblocked set of alternatives will have some consequences under the last axiom below, see also Remark 3.1. We call $b_{H}: P_{0}(A) \rightarrow\{1, \ldots, n+1\}$ the blocking function of $H$.

We consider the following axioms on $H$.

Anonymity For all $R^{N} \in L^{N}$ and for all permutations $\pi$ of $N, H\left(R^{1}, \ldots, R^{n}\right)=H\left(R^{\pi(1)}, \ldots, R^{\pi(n)}\right)$.

Maskin monotonicity For all $R^{N}=\left(R^{1}, \ldots, R^{n}\right), Q^{N}=\left(Q^{1}, \ldots, Q^{n}\right) \in L^{N}$, and $x \in H\left(Q^{N}\right)$, if $x Q^{i} y$ implies $x R^{i} y$ for all $y \in A$ and $i \in N$, then $x \in H\left(R^{N}\right)$.

Independent blocking The blocking function $b_{H}$ is additive, i.e., $b_{H}\left(B_{1} \cup B_{2}\right)=b_{H}\left(B_{1}\right)+b_{H}\left(B_{2}\right)$ for all $B_{1}, B_{2} \in P_{0}(A)$ with $B_{1} \cap B_{2}=\varnothing$.

Anonymity needs no further explanation. Maskin monotonicity says that if a chosen alternative does not gets worse in any one's preference, then it remains to be chosen. It is a necessary condition for implementation in Nash equilibrium (Maskin, 1999). For later reference we also formulate the following weaker version of monotonicity. In this weaker version

\footnotetext{
${ }^{3}$ In the next section we formulate this choice as an additional axiom.
} 
we require that if an alternative $x$ is shifted upwards in some preference, then the preference among all the other alternatives does not change. Note that in the premiss of Maskin monotonicity preferences among the other alternatives may change, as long as $x$ is shifted upwards.

Monotonicity For all $R^{N}=\left(R^{1}, \ldots, R^{n}\right), Q^{N}=\left(Q^{1}, \ldots, Q^{n}\right) \in L^{N}$, and $x \in H\left(Q^{N}\right)$, if $x Q^{i} y \Rightarrow x R^{i} y$ and $z R^{i} y \Leftrightarrow z Q^{i} y$ for all $y, z \in A \backslash\{x\}$ and $i \in N$, then $x \in H\left(R^{N}\right)$ and $H\left(R^{N}\right) \subseteq H\left(Q^{N}\right)$.

That monotonicity is weaker than Maskin monotonicity can be seen as follows. It is sufficient to consider $Q^{N}$ and $R^{N}$ in the statement of monotonicity such that $R^{N}$ arises from $Q^{N}$ by switching alternative $x$ with the alternative right above $x$, say $y$, for just one voter. By Maskin monotonicity, $x \in H\left(R^{N}\right)$. Suppose there is an alternative $z \in A \backslash\{x\}$ with $z \in H\left(R^{N}\right)$. By Maskin monotonicity again, now applied to $z$, we obtain $z \in H\left(Q^{N}\right)$. Thus, $H\left(R^{N}\right) \subseteq H\left(Q^{N}\right)$.

Under anonymity, independent blocking implies for instance that if $S_{1}$ minimally blocks $B_{1}$ and $S_{2}$ minimally blocks $B_{2}$, and $S_{1}$ and $S_{2}$ as well as $B_{1}$ and $B_{2}$ are disjoint, then $S_{1} \cup S_{2}$ minimally blocks $B_{1} \cup B_{2}$. Observe, here, that by definition $S_{1} \cup S_{2}$ blocks $B_{1} \cup B_{2}$; the contribution of the independent blocking condition is the addition of the word 'minimal', so no smaller coalition can block $B_{1} \cup B_{2}$.

Remark 3.1. By the choice $b_{H}(A)=n+1$, independent blocking implies the equivalence $b_{H}(B)=n+1 \Leftrightarrow B=A$. Namely, suppose $b_{H}(B)=n+1$ for some $\varnothing \neq B \subsetneq A$. Then $b_{H}(A)=b_{H}(B)+b_{H}(A \backslash B)$ implies that $b_{H}(A \backslash B)=0$, a contradiction since $b_{H}(A \backslash B) \geq 1$ by definition. It also implies that $H$ is non-imposed, i.e., for every $a \in A$ there is a profile $R^{N} \in L^{N}$ such that $H\left(R^{N}\right)=\{a\}$. To see this, suppose that there is some $a \in A$ such that $H\left(R^{N}\right) \neq\{a\}$ for all $R^{N} \in L^{N}$. Then $b_{H}(A \backslash\{a\})=n+1$, so independent blocking implies $b_{H}(\{a\})=b_{H}(A)-b_{H}(A \backslash\{a\})=0$, a contradiction.

The following theorem is the announced characterization of the social choice correspondences $M_{\beta}$.

Theorem 3.2. Let $n+1 \geq m$ and let $H$ be an SCC. The following statements are equivalent.

(1) H satisfies anonymity, Maskin monotonicity, and independent blocking.

(2) There is a function $\beta: A \rightarrow \mathbb{N}$ with $\sum_{x \in A} \beta(x)=n+1$ such that $H=M_{\beta}$.

In the proof of Theorem 3.2 we use the following lemmas, starting with a proof of Maskin monotonicity of $M_{\beta}{ }^{4}$

Lemma 3.3. Let $\beta: A \rightarrow \mathbb{N}$ such that $\sum_{x \in A} \beta(x)=n+1$. Then $M_{\beta}$ is Maskin monotonic.

Proof. Let $Q^{N}$ and $R^{N}$ be as in the definition of Maskin monotonicity, and $x \in M_{\beta}\left(Q^{N}\right)$. Without loss of generality we assume that there is a voter $v$ such that $Q^{N \backslash\{v\}}=R^{N \backslash\{v\}}$. Let $f^{*}=\left(x_{1}, C_{1} ; \ldots ; x_{m-1}, C_{m-1} ; x\right)$ be an f.e.p. for $Q^{N}$, where $A=\left\{x_{1}, \ldots, x_{m-1}, x\right\}$. If $v \notin C_{1} \cup \ldots \cup C_{m-1}$ then it is easy to see that $f^{*}$ is still an f.e.p. for $R^{N}$, so that $x \in M_{\beta}\left(R^{N}\right)$. Now assume $v \in C_{1} \cup \ldots \cup C_{m-1}$. If $v \in C_{j}$ with $j>1$, then we may eliminate $x_{1}, \ldots, x_{j-1}$ and all voters in $C_{1} \cup \ldots \cup C_{j-1}$ first, and next continue the argument with the remaining profile, where now all voters in $C_{j}$ have $x_{j}$ bottom ranked according to $\mathrm{Q}_{j}^{C}$. So, without loss of generality, let $v \in C_{1}$.

The rest of the proof is based on a three step algorithm.

Step 1If the bottom alternative of $R^{v}$ is equal to $x_{1}$, then $f^{*}$ is still an f.e.p. for $R^{N}$ and we are done. Otherwise, go to Step 2 .

Step 2Let the bottom alternative of $R^{v}$ be $x_{\ell} \neq x_{1}$, so $\ell \in\{2, \ldots, m-1\}$. If all voters in $C_{\ell}$ have $x_{\ell}$ as bottom alternative in $R^{N}$, then we can first eliminate $x_{\ell}$ via $C_{\ell}$ and go back to Step 1 for the reduced profile. Otherwise, go to Step 3.

Step 3Take $\hat{v} \in C_{\ell}$ with $x_{\ell}$ not as bottom alternative and note that the bottom alternative of $R^{\hat{v}}=Q^{\hat{v}}$ is some $x_{j}$ with $j<\ell$ (since $x_{j}$ must be eliminated before $x_{\ell}$ in $\left.f^{*}\right)$. Then modify $C_{\ell}$ to $\hat{C}_{\ell}=\left(C_{\ell} \cup\{v\}\right) \backslash\{\hat{v}\}$ and modify $C_{1}$ to $\hat{C}_{1}=\left(C_{1} \cup\{\hat{v}\}\right) \backslash\{v\}$. (In words, we switch $v$ and $\hat{v}$.) Go back to Step 1.

Repeat this procedure until the final substitute of $v$ in the modified $C_{1}$ has $x_{1}$ at bottom. Then we can apply an f.e.p. resulting in $x$, so that $x \in M_{\beta}\left(R^{N}\right)$.

In what follows we use the notation $\beta(B)$ for $\sum_{x \in B} \beta(x)$, where $B \in P_{0}(A)$.

Lemma 3.4. Let $\beta: A \rightarrow \mathbb{N}$ such that $\sum_{x \in A} \beta(x)=n+1$. Let $B \in P_{0}(A)$ and $S \in P_{0}(N)$. Then $S$ minimally blocks $B$ at $M_{\beta}$ if and only if $|S|=\beta(B)$.

Proof. For the only-if statement, suppose that $S$ minimally blocks $B$ at $M_{\beta}$. Let $Q^{S} \in L^{S}$ such that $x Q^{i} y$ for all $i \in S, x \in A \backslash B$ and $y \in B$. Then $M_{\beta}\left(Q^{S}, R^{N \backslash S}\right) \subseteq A \backslash B$ for all $R^{N \backslash S} \in L^{N \backslash S}$. Consider, in particular, a profile $\tilde{R}^{N \backslash S}$ such that $x \tilde{R}^{i} y$ for all $x \in B, y \in A \backslash B$, and $i \in N \backslash S$. For an f.e.p. $\left(x_{1}, C_{1} ; \ldots ; x_{m-1}, C_{m-1} ; x_{m}\right)$ for $\left(Q^{S}, \tilde{R}^{N \backslash S}\right)$ we have $x_{m} \in M_{\beta}\left(Q^{S}, \tilde{R}^{N \backslash S}\right) \subseteq A \backslash B$ and therefore $B \subseteq\left\{x_{1}, \ldots, x_{m-1}\right\}$. By definition of $\tilde{R}^{N \backslash S}$ we must have $C_{j} \subseteq S$ for every $j \in\{1, \ldots, m-1\}$ with $x_{j} \in B$. Therefore, $|S| \geq \sum_{j \in\{1, \ldots, m-1\}: x_{j} \in B}\left|C_{j}\right|=\beta(B)$. Now consider any coalition $T \subseteq S$ such that $|T|=\beta(B)$ and a profile $P^{T} \in L^{T}$ such that $x P^{i} y$ for all $x \in A \backslash B$ and $y \in B$. Then $|N \backslash T|=n-\beta(B)=\beta(A \backslash B)-1<\beta(A \backslash B)$, so that any f.e.p. for any $\left(P^{T}, R^{N \backslash T}\right)$ results in an alternative of $A \backslash B$. Hence, $T$ blocks $B$ at $M_{\beta}$, so that $T=S$ by minimality of $S$. Hence, $|S|=\beta(B)$.

${ }^{4}$ The current proof is a special case of the proof in Peleg and Peters (2015), Lemma 3.7, and is included here for completeness. 
For the if-statement, if $|S|=\beta(B)$ then by the preceding argument $S$ blocks $B$ at $M_{\beta}$. For any $T \subseteq S$ to block $B$ we need $|T| \geq \beta(B)$ by the same argument as in the first part of the proof. Hence, $S$ minimally blocks $B$ at $M_{\beta}$.

The next lemma is crucial for proving the implication $(i) \Rightarrow$ (ii) in Theorem 3.2. ${ }^{5}$

Lemma 3.5. Let $\beta: A \rightarrow \mathbb{N}$ such that $\sum_{x \in A} \beta(x)=n+1$. Let $x \in$ Aand $R^{N} \in L^{N}$. The following statements are equivalent.

(i) $x \in M_{\beta}\left(R^{N}\right)$.

(ii) There are no $S \in P_{0}(N)$ and $B \in P_{0}(A)$ such that $|S| \geq \beta(A \backslash B), x \in A \backslash B$, and $y R^{i} x$ for all $i \in S$ and $y \in B$.

Proof. For the implication ( $i) \Rightarrow(i i)$, let $x \in M_{\beta}\left(R^{N}\right)$ and let $\left(x_{1}, C_{1} ; \ldots ; x_{m-1}, C_{m-1} ; x\right)$ be an f.e.p. for $R^{N}$. Suppose there were $S$ and $B$ as in (ii). Write $B=\left\{x_{i_{1}}, \ldots, x_{i_{|B|}}\right\} \subseteq\left\{x_{1}, \ldots, x_{m-1}\right\}$, then $\left(\cup_{j=1}^{|B|} C_{i_{j}}\right) \cap S=\varnothing$ by definition of an f.e.p., and $\left|\cup \cup_{j=1}^{|B|} C_{i_{j}}\right|=\beta(B)$. Hence $|S|+\left|\cup_{j=1}^{|B|} C_{i_{j}}\right| \geq \beta(A \backslash B)+\beta(B)=n+1$, a contradiction.

We prove the implication (ii) $\Rightarrow$ (i) by induction on the number of alternatives $m$. Assume that (ii) holds.

If $m=2$, say $A=\{x, y\}$, then there is no $S \in P_{0}(N)$ such that $|S| \geq \beta(x)$ and $y R^{i} x$ for all $i \in S$, so that $M_{\beta}\left(R^{N}\right)=\{x\}$.

Now suppose that $m>2$ and that the implication (ii) $\Rightarrow$ (i) holds if there are less than $m$ alternatives. For every $B \in P_{0}(A \backslash\{x\})$ denote $S_{B}=\left\{i \in N: y R^{i} x\right.$ for all $\left.y \in B\right\}$. Then (ii) is equivalent to

$$
\left|S_{B}\right|<\beta(A \backslash B) \text { for allB } \in P_{0}(A \backslash\{x\})
$$

hence to

$$
\left|N \backslash S_{B}\right| \geq \beta(B) \text { for allB } \in P_{0}(A \backslash\{x\}) .
$$

We consider two cases.

Case 1There exists $\tilde{B} \in P_{0}(A \backslash\{x\})$ with $|\tilde{B}| \leq m-2$ and $\left|N \backslash S_{\tilde{B}}\right|=\beta(\tilde{B})$.

For this case we consider the two following subproblems:

- $N_{1}=N \backslash S_{\tilde{B}}, A_{1}=\tilde{B} \cup\{x\}, \beta_{1}(y)=\beta(y)$ for all $y \in \tilde{B}, \beta_{1}(x)=1$, and $R_{1}^{i}=R_{\mid A_{1}}^{i}$ for all $i \in N_{1}{ }^{6}$

- $N_{2}=S_{\tilde{B}}, A_{2}=A \backslash \tilde{B}, \beta_{2}(y)=\beta(y)$ for all $y \in A_{2}$, and $R_{2}^{i}=R_{\mid A_{2}}^{i}$ for all $i \in N_{2}$.

We next show that (1) holds for the first subproblem. If not, then there is a $B \in P_{0}(\tilde{B})$ such that $|T| \geq \beta_{1}\left(A_{1} \backslash B\right)$, where $T=\left\{i \in N_{1}: y R_{1}^{i} x\right.$ for ally $\left.\in B\right\}$. Then $\left|T \cup S_{\tilde{B}}\right|=|T|+\left|S_{\tilde{B}}\right| \geq\left[\beta_{1}(x)+\beta(\tilde{B})-\beta(B)\right]+[n-\beta(\tilde{B})]=\beta(A \backslash B)$, hence $\left|S_{B}\right| \geq \beta(A \backslash B)$, which is a violation of (1) for the original problem. Therefore, (1) must hold for the first subproblem, implying that $x \in M_{\beta_{1}}\left(R_{1}^{N_{1}}\right)$ by induction.

Similarly, suppose that (1) does not hold for the second subproblem. Then there is a $B \in P_{0}(A \backslash(\tilde{B} \cup\{x\}))$ such that $|T| \geq \beta_{2}\left(A_{2} \backslash B\right)$, where $T=\left\{i \in S_{\tilde{B}}: y R_{2}^{i} x\right.$ for ally $\left.\in B\right\}$. Then $\left|T \cup\left(N \backslash S_{\tilde{B}}\right)\right|=|T|+\left|N \backslash S_{\tilde{B}}\right| \geq[\beta(A)-\beta(B)-\beta(\tilde{B})]+\beta(\tilde{B})=\beta(A \backslash B)$, which is a violation of (1) for the original problem. We conclude that (1) must hold for the second subproblem as well, so that $x \in M_{\beta_{2}}\left(R_{2}^{N_{2}}\right)$ by induction.

Now let $\left(z_{1}, C_{1} ; \ldots ; z_{|\tilde{B}|}, C_{|\tilde{B}|} ; x\right)$ be an f.e.p. for the first subproblem and let $\left(u_{1}, D_{1} ; \ldots ; u_{m-1-|\tilde{B}|}, D_{m-1-|\tilde{B}|} ; x\right)$ be an f.e.p. for the second subproblem. Since, in particular, $y R^{i} x$ for all $y \in \tilde{B}$ and $i \in N_{2}=S_{\tilde{B}}$, it follows that

$$
\left(u_{1}, D_{1} ; \ldots ; u_{m-1-|\tilde{B}|}, D_{m-1-|\tilde{B}|} ; z_{1}, C_{1} ; \ldots ; z_{|\tilde{B}|}, C_{|\tilde{B}|} ; x\right)
$$

is an f.e.p. for the original problem, implying that in this case we have $x \in M_{\beta}\left(R^{N}\right)$.

Case 2 For all $\tilde{B} \in P_{0}(A \backslash\{x\})$ with $|\tilde{B}| \leq m-2$ we have $\left|N \backslash S_{\tilde{B}}\right|>\beta(\tilde{B})$.

Suppose there is an $\ell \in N$ such that $x$ is not ranked at the last or second last position in $R^{\ell}$, and let $\widehat{y}$ be the alternative ranked right below $x$. We switch $x$ and $\widehat{y}$ in voter $\ell$ 's preference to obtain a new preference $\widehat{R}^{\ell}$ and a new preference profile $\widehat{R}^{N}=\left(R^{1}, \ldots, R^{\ell-1}, \widehat{R}^{\ell}, R^{\ell+1}, \ldots, R^{N}\right)$ that still satisfies (2): for any set $B$ with $|B| \leq m-2$ this holds because of the strict inequality in Case 2, and for $B=A \backslash\{x\}$ this holds since $x$ is not ranked last in $\widehat{R}^{\ell}$.

If Case 1 applies to $\widehat{R}^{N}$, then $x \in M_{\beta}\left(\widehat{R}^{N}\right)$. Thus, by Lemma 3.3, $x \in M_{\beta}\left(R^{N}\right){ }^{7}$ If Case 1 does not apply to $\widehat{R}^{N}$, then we repeat this step for some voter $\ell^{\prime} \in N$ with $x$ not ranked last or second last at $\widehat{R}^{\ell^{\prime}}$, and so on, until either Case 1 applies or there is no voter left with $x$ not ranked at the last or second last position.

In the latter case, we have a profile, say $\tilde{R}^{N}$, for which still (2) holds and with $x$ ranked last or second last for each voter $i \in N$. Observe that $y$ is last ranked for all voters in $N \backslash S_{\{y\}}$ for all $y \in A \backslash\{x\}$. Also, by (2), $\left|N \backslash S_{\{y\}}\right| \geq \beta(y)$ for all $y \in A \backslash\{x\}$. It follows

\footnotetext{
${ }^{5}$ The lemma is a reformulation of an existing result: see Theorem 9.3.6 in Peleg and Peters (2010), which in turn goes back to a result of Polishchuk (1978).

${ }^{6} R_{\mid B}^{i}$ denotes the restriction of $R^{i}$ to $B$

7 In fact, monotonicity would be sufficient for this argument.
} 
that in any f.e.p. for $\tilde{R}^{N}$ every $y \in A \backslash\{x\}$ is bottom ranked by at least $\beta(y)$ voters and therefore eliminated, so that $M_{\beta}\left(\tilde{R}^{N}\right)=\{x\}$. By Lemma 3.3 again, $x \in M\left(R^{N}\right)$.

By (2), Cases 1 and 2 are exhaustive, which completes the proof of the lemma.

We are now sufficiently equipped to prove the main result of the paper.

Proof of Theorem 3.2. We first prove the implication (ii) $\Rightarrow$ (i).

Let $\beta: A \rightarrow \mathbb{N}$ such that $\sum_{x \in A} \beta(x)=n+1$. Clearly, $M_{\beta}$ is anonymous. Maskin monotonicity of $M_{\beta}$ follows from Lemma 3.3. From Lemma 3.4 it follows that the blocking function $b=b_{M_{\beta}}$ of $M_{\beta}$ is $\beta$, i.e., $b(B)=\beta(B)$ for all $B \in P_{0}(A)$, and independent blocking follows since $\beta$ is additive.

For the implication $(i) \Rightarrow($ ii $)$, let $H$ satisfy the three axioms. Define $\beta: A \rightarrow \mathbb{N}$ by $\beta(x)=b_{H}(x)$ for all $x \in A$. Then by independent blocking, $\sum_{x \in A} \beta(x)=n+1$, so $M_{\beta}$ is well-defined. We prove that $H=M_{\beta}$. Let $R^{N} \in L^{N}$.

First, let $x \in M_{\beta}\left(R^{N}\right)$. Then there exists an f.e.p. $\left(x_{1}, C_{1} ; \ldots ; x_{m-1}, C_{m-1} ; x\right)$ with respect to $R^{N}$. Let now $Q^{N}$ be the profile that is obtained from $R^{N}$ by lowering $x_{j}$ to the bottom of $R^{i}$ for all $i \in C_{j}$ and for $j=1, \ldots, m-1$, and leaving everything else intact. By the definition of blocking coefficients, $H\left(Q^{N}\right) \subseteq A \backslash\left\{x_{j}\right\}$ for all $j=1, \ldots, m-1$, so that $H\left(Q^{N}\right)=\{x\}$. Finally, since $x R^{i} x_{j}$ for all $i \in C_{j}$ and $j=1, \ldots, m-1$ (by the third condition in Definition 2.1), and since $H$ is Maskin monotonic, $x \in H\left(R^{N}\right)$.

Second, let $x \in H\left(R^{N}\right)$. Suppose there are $S \in P_{0}(N)$ and $B \in P_{0}(A)$ as in (ii) of Lemma 3.5, i.e., $|S| \geq \beta(A \backslash B), x \in A \backslash B$, and $y R^{i} x$ for all $y \in B$. For each $i \in S$ let $Q^{i} \in L$ be a preference with $y Q^{i} z \Leftrightarrow y R^{i} z$ for all $y, z \in A \backslash B$ and with $y Q^{i} z$ for all $y \in B$ and $z \in A \backslash B$. Since $|S| \geq \beta(A \backslash B)=b_{H}(A \backslash B)$, by anonymity we have that $S$ blocks $A \backslash B$ at $H$, so that $x \notin H\left(Q^{S}, R^{N \backslash S}\right)$. On the other hand, since $x \in H\left(R^{N}\right)$ and $y R^{i} x$ for all $i \in S$ and $y \in B$, Maskin monotonicity of $H$ implies $x \in H\left(Q^{S}, R^{N \backslash S}\right)$, a contradiction. Hence, $x \in M_{\beta}\left(R^{N}\right)$ by Lemma 3.5.

We next show that the three axioms in Theorem 3.2 are logically independent, by exhibiting three examples.

Example 3.6. Let $A=\{x, y\}$ and let $N=\{1,2,3,4\}$. Define the SCC $H$ as follows. Let $R^{N} \in L^{N}$. If $R^{1}=R^{2}=y x$ (i.e., both 1 and 2 prefer $y$ above $x$ ) then $H\left(R^{N}\right)=\{y\}$. If $R^{2}=R^{3}=R^{4}=x y$ then $H\left(R^{N}\right)=\{x\}$. In all other cases, $H\left(R^{N}\right)=\{x, y\}$. Then $H$ is Maskin monotonic and independently blocking: $b_{H}(x)+b_{H}(y)=3+2=5=b_{H}(A)$. However, $H$ is not anonymous.

Example 3.7. It is not difficult to see that there exist anonymous selections from $M_{\beta}$ : e.g., take $V \in L$ and let $F\left(R^{N}\right)$ be the alternative of $M_{\beta}\left(R^{N}\right)$ that is maximal according to $V$. Such a selection is still independently blocking. However, it cannot be Maskin monotonic (if $m \geq 3$ ) because then by Muller and Satterthwaite (1977) it would be dictatorial. It is not hard to see that $F$ is still monotonic, as follows. Suppose that $R^{N}$ and $Q^{N}$ are as in the definition of monotonicity, that is, $R^{N}$ arises from $Q^{N}$ by shifting $x$ upwards, leaving everything else intact. Suppose that $\{x\}=F\left(Q^{N}\right)$, hence $x$ is the $V$-maximal element of $M_{\beta}\left(Q^{N}\right)$. Then by monotonicity of $M_{\beta}$ we have $x \in M_{\beta}\left(R^{N}\right) \subseteq M_{\beta}\left(Q^{N}\right)$. This implies that $x$ is still the $V$-maximal element of $M_{\beta}\left(R^{N}\right)$, hence $F\left(R^{N}\right)=\{x\}$. We conclude that Maskin monotonicity in Theorem 3.2 cannot be weakened to monotonicity.

Example 3.8. The Pareto correspondence $P$ assigns to each profile $R^{N}$ the set $\left\{x \in A\right.$ : there is no $y \in A$ with $y P^{i} x$ for all $\left.i \in N\right\}$. It is anonymous and Maskin monotonic, but it is not independently blocking: $b_{P}(B)=n$ for every $B \in P_{0}(A), B \neq A$.

We conclude this section by discussing the relation with the majority rule for the case $m=2$, say $A=\{a, b\}$. In the formulation of May (1952), the majority rule is a social welfare function which ranks $x \in\{a, b\}$ above $y \in\{a, b\}$ if and only if a strict majority of voters ranks $x$ above $y$. In case of a tie, the rule assigns indifference between $a$ and $b$. May (1952) characterizes this rule by four conditions: single-valuedness, anonymity, neutrality, and Maskin monotonicity (which takes a simple form in this case).

In our framework, if $m=2$ and the number of voters is odd, then majority rule is equivalent to the (single-valued) correspondence $M_{\beta}$ with $\beta(a)=\beta(b)$. If the number of voters is even, then closest to majority rule is $M_{\beta}$ (again single-valued) with either $\beta(a)=\beta(b)+1$ or $\beta(a)=\beta(b)-1$ - we need to break ties. For $m=2$, our axioms of anonymity, Maskin monotonicity, and independent blocking are still independent: see Examples 3.6, 3.8, and for the independence of Maskin monotonicity take minority rule with tie-breaking. These three axioms characterize a larger set of rules, namely for all allowed choices of weights. Consider the following condition, formulated here for general $m$ and a correspondence $H$ with blocking function $b_{H}$ :

Weak neutrality For all $B, B^{\prime} \in P_{0}(A)$ with $|B|=\left|B^{\prime}\right|,\left|b_{H}(B)-b_{H}\left(B^{\prime}\right)\right| \leq|B|$.

For the case $m=2$, adding this condition to anonymity, Maskin monotonicity, and independent blocking, returns exactly the majority rule if the number of voters is odd, and the two rules above if the number of voters is even. By using the same three examples, it follows that the other three conditions are still logically independent if weak neutrality is added. If $m>2$ then the additional condition of weak neutrality selects the correspondences $M_{\beta}$ which are as neutral as possible. Thus, particularly in the axiomatic sense, feasible eliminations procedures can be seen as one possible extension of majority rule to the case of more than two alternatives. 


\section{Conclusion}

For any sincere preference profile, in the game associated with an exactly and strongly consistent social choice function there exists always a strong Nash equilibrium resulting in the sincere outcome. Also, for such a social choice function, sincere voting itself is a safe strategy in the sense that a coalition deviating from it is not guaranteed a better outcome. In order to construct exactly and strongly consistent social choice functions feasible elimination procedures play a central role. Moreover, voting systems based on feasible elimination procedures are of practical relevance as well. In this paper, we have provided an axiomatic characterization of the social choice correspondence that assigns maximal alternatives, i.e., alternatives resulting from feasible elimination procedures. The proof of our axiomatic characterization is closely related to earlier results, in particular Theorem 9.3.6 in Peleg and Peters (2010), which, in turn, goes back to an unpublished result of Polishchuk (1978). This theorem is phrased in terms of an effectivity function and its core.

\section{References}

Abdou, J., 1989. Convexity of integer veto and elimination procedures. Social. Choice Welf. 6, 63-70.

Balinski, M., Laraki, R., 2010. Majority Judgment: measuring, Ranking, and Electing. The MIT Press, Cambridge, Massachusetts, London UK. Bartholdi III, J.J., Tovey, C.A., Trick, M.A., 1989. The computational difficulty of manipulating an election. Social. Choice Welf. 6, $227-241$.

Danilov, V.I., Sotskov, A.I., 2002. Social Choice Mechanisms. Springer, Berlin Heidelberg.

Dutta, B., 1983. Further results on voting with veto. In: Pattanaik, P.K., Salles, M. (Eds.), Social Choice and Welfare, North-Holland, Amsterdam, pp. 239-250. Gibbard, A., 1973. Manipulation of voting schemes: a general result. Econometrica 41, 587-602.

Holzman, R., 1986. On strong representations of games by social choice functions. J. Math. Econ. 15, 39-57.

Maskin, E., 1999. Nash equilibrium and welfare optimality. Rev. Econ. Stud. 66, 23-38.

May, K.O., 1952. A set of independent necessary and sufficient conditions for simple majority decision. Econometrica 20, 680-684.

Moulin, 1980. Implementing efficient, anonymous and neutral social choice functions. J. Math. Econ. 7, 249-264.

Moulin, H, 1982. Voting with proportional veto power. Econometrica 50, 145-162.

Muller, E., Satterthwaite, M.A., 1977. The equivalence of strong positive association and strategy-proofness. J. Econ. Theory 14, 412-418.

Peleg, B., 1978. Consistent voting systems. Econometrica 46, 153-161.

Peleg, B., Peters, H., 2010. Strategic Social Choice. Springer, Berlin Heidelberg.

Peleg, B., Peters, H., 2015. Choosing $k$ from $m$ : feasible elimination procedures reconsidered. Working Paper, Maastricht University, The Netherlands.

Peleg, B., Procaccia, AD., 2007. Mediators enable truthful voting. DP 451, Center for the Study of Rationality. The Hebrew University of Jerusalem.

Polishchuk, I., 1978. Monotonicity and uniqueness of consistent voting systems. Center for Research in Mathematical Economics and Game Theory, Hebrew University of Jerusalem.

Satterthwaite, M.A., 1975. Strategy-proofness and arrow's. J. Econ. Theory 10, 187-207. 